\title{
3 Psychotherapie und Bewusstsein
}

\author{
Joachim Galuska
}

Psychotherapie verstehe ich als Veränderung des Bewusstseins eines Patienten oder einer Patientin durch das Bewusstsein von Therapeuten mit dem Ziel, zu lernen das eigene Leben in Verbundenheit mit der mitmenschlichen Gemeinschaft so zu gestalten, wie es diesem Menschen entspricht. Konventionelle Psychotherapie reflektiert dabei nicht primär den Bewusstseinszustand des Therapeuten, sondern richtet sich vorwiegend auf die Patienten, ihr Erleben und Verhalten, unter theoriegeleiteten Gesichtspunkten. Der konventionelle Bewusstseinszustand, aus dem heraus therapeutisch gehandelt wird und der als Grundlage für die gemeinsame therapeutische Arbeit mit den Patienten dient, ist in der Regel das Ich-Bewusstsein.

\section{Ich-Identität}

Die Ich-Entwicklung ist, wie uns Ken Wilber (1996) gezeigt hat, ein Teil einer umfassenderen Bewusstseinsentwicklung, die er in Form einer Reihe von aufeinanderfolgenden Stufen oder Wellen der Struktur unseres Bewusstseins beschreibt. In ihnen unterscheiden sich jeweils das gesamte Selbstverständnis, unsere Beziehung zur Welt, die kognitiven Funktionen, die Gefühlswelt, das moralische Empfinden, das spirituelle Erleben usw. Das bei Erwachsenen vorherrschende Ich-Bewusstsein oder personale Bewusstsein entwickelt sich aus sogenannten präpersonalen Stufen, in denen magisches, mythisches und vorrationales Denken noch vorherrschen. Im Einklang mit der tiefenpsychologischen Entwicklungspsychologie und Identitätstheorie kann man sagen, dass das bei Erwachsenen vorherrschende Bewusstsein im Wesentlichen Ich-Bewusstsein ist. Es ist begründet und bewirkt durch den Identifizierungsprozess, der im Zentrum der sogenannten Ich-Struktur oder Ich-Organisation steht. Die Identifizierung bewirkt letztlich die Ich-Identität, also ein sta- 
biles zusammenhängendes Konzept von uns selbst. Als Ich erfahre ich mich, wenn ich mich ständig mit meinem Erleben identifiziere. Somit ist das Gefühl „,ich“ zu sein eigentlich ein permanenter Prozess des Sich-Identifizierens mit Gedanken, Empfindungen, Impulsen, Gefühlen und Wahrnehmungen. Aus der Fülle unserer Erlebnisse, mit denen wir uns identifizieren und an die wir uns erinnern, entwickeln wir offenbar ein Konzept von uns selbst. Es besteht aus Vorstellungen und Gefühlen darüber, wer ich bin, wie ich mich anfühle, verhalte, mit anderen Menschen umgehe. Dieses Konzept ist somit eine mentale und psychische Konstruktion, eben ein Konzept, so etwas wie ein inneres Modell. Ständig muss ich es neu erschaffen, arbeite es weiter aus, vergewissere mich seiner. Aus unseren Erfahrungen abstrahieren wir beständig, bilden Konzepte über uns selbst, die anderen Menschen und die Welt. Erst durch den Identifizierungsprozess - das bin ich, das ist ein anderer, das ist ein Gegenstand - entsteht unser alltägliches Selbstverständnis und Weltverständnis. Meine Innen- und meine Außenwelt besteht dann in der Folge aus konzeptualisierten Objekten. Es geht nicht so sehr um mein Denken oder Fühlen, sondern um meine Gedanken und meine Gefühle. Andere Menschen sind Objekte meiner Begierden und Interessen. Eine sogenannte „Objekt-Beziehungs-Theorie“ von Kernberg beschreibt die verschiedenen Identifizierungsprozesse hin zur Ich-Identität.

„Die Ich-Identität ist die umfassende Strukturierung von Identifizierungen und Introjektionen unter dem steuernden Prinzip der synthetischen Funktion des Ich“,

schreibt Kernberg (1981). Anschaulicher sagt es vielleicht Karl Popper:

„Das Ich ist nicht nur ein ,reines Ich", d.h. ein loses Subjekt, es ist vielmehr unglaublich reich. Wie ein Steuermann beobachtet und handelt es gleichzeitig. Es ist tätig und erleidend, erinnert sich der Vergangenheit und plant und programmiert die Zukunft; es ist in Erwartung und disponiert. Es enthält in rascher Abfolge oder mit einem Mal Wünsche, Pläne, Hoffnungen, Handlungsentscheidungen und ein lebhaftes Bewusstsein davon, ein handelndes Ich zu sein, ein Zentrum der Aktion. Und esverdankt diese Ichheit weitgehend der Wechselwirkung mit anderen Personen, mit dem Ich anderer, und derWelt 3“, der Kultur (Popper zitiert in Hinterhuber, 2001, S. 149).

Das reife Ich steht also im Zentrum unserer Wahrnehmung und Handlung. „Ich denke, also bin ich“, ist nach Descartes der Ausgangspunkt aller Philosophie. „Ich denke“, identifiziere mich also mit meinem eigenen Denken, das ich als meines betrachte. „Also bin ich“, diese Identifizierung mit meinem Denken erschafft meine Existenz, gibt mir erst Substanz. Ich bin also die Folge meiner Gedanken und meines Denkens. Und dies ist nicht statisch zu verstehen, als etwas Gegebenes, sondern als ein Prozess, der sich beständig wiederholt und mein Selbstverständnis und mein Weltverständnis validieren muss, wie die Ich-Psychologen und Objekt-BeziehungsTheoretiker uns gezeigt haben.

Mein Leben lang muss ich also erkannt werden, anerkannt werden, gesehen und bestätigt werden, als der, für den ich mich halte, und muss mich meiner selbst und meiner Vorstellungen über meine Mitmenschen vergewissern.

Im Grunde besitzt die ausgereifte Ich-Struktur zwei Aspekte:

- die Entfaltung der Individualität und

- der Bezogenheit. 
Individualität bedeutet Selbstverwirklichung, Autonomie, Authentizität, Fähigkeit zu schöpferischem und kreativem Handeln. Die humanistische Psychologie betont auch die Entwicklung von Lebensfreude und Lebensgenuss, innerer Schönheit und innerem Reichtum. Sie fordert eine Aufgabe der Opferperspektive, eine Rücknahme der Projektion der Verantwortung für die eigene Entwicklung auf die äußeren Bedingungen oder die eigene Biographie und eine Entwicklung hin zur Übernahme vollständiger Verantwortung für die eigene Lebensgestaltung, für die eigenen Antworten auf die Bedingungen, in denen wir leben.

Bezogenheit meint die Fähigkeit zum Du, zur Begegnung, zum Dialog, damit also zur Wahrnehmung des anderen Menschen als ebenso ein Ich, wie ich es bin, damit also als ein Subjekt dieser Inter-Subjektivität. Dies ist die Voraussetzung zur Partizipation am Wir, an der Gemeinschaft, zur Übernahme von Mitverantwortung und damit zur Bildung von reifen Paarbeziehungen und reifen Familienstrukturen. Damit wird das eigene Leben auch anerkannt in seiner Bezogenheit auf den historischen, kulturellen und gesellschaftlichen Kontext und gelebt in engagierter Verantwortung, Solidarität und Sorge für die Mitmenschen und die Natur (Petzold 1993). Selbstgestaltung und Weltgestaltung sind wesentliche Herausforderungen der IchStruktur.

\section{Konventionelle und beseelte Psychotherapie}

Konventionelle Psychotherapie basiert in der Regel auf der Ich-Struktur und dem IchBewusstsein eines Therapeuten und den damit verbundenen Identifizierungen mit dem Menschenbild und den Konzeptionen seiner psychotherapeutischen Ausbildung. So hilfreich die entsprechende Einflussnahme auf die Patienten auch sein mag, ein solches Handeln ist letztendlich konzeptionell gefangen. Wir behandeln auf diese Weise nicht die Krankheiten oder Störungen unserer Patienten, sondern nur unsere Vorstellungen davon, unsere Konzepte. In unserer Diagnostik filtern wir gemäß unseres Störungskonzeptes die entsprechenden Aspekte heraus, erklären dies unseren Patienten und intervenieren dann gemäß unserer Theorie. So bringen wir den Patienten unser Störungsverständnis bei. Die Patienten ersetzen also ihr offenbar untaugliches Modell von sich selbst durch das Modell des Therapeuten. Da unsere schulenspezifischen Modelle wahrscheinlich besser sind als die unserer Patienten, funktioniert dies auch ein wenig. Vielleicht liegt es aber auch daran, dass nach Therapiestudien nur 15-30\% der Wirksamkeit auf die Methode zurückzuführen sind, der Rest auf die therapeutische Beziehung und andere Faktoren. So übertragen wir aber auf die Patienten ein ideologisch geprägtes einseitiges reduziertes Menschenbild. Wir betrachten sie nicht als lebendige Menschen, als Wunder der Schöpfung, als verirrte Seele denen wir beistehen können, zu heilen und zu ihrem Leben zu finden. Das Problem ist noch nicht einmal die Perspektive, der Blickwinkel, sondern die Ideologisierung. Wir sind gefangen in unseren Perspektiven und werden aggressiv, wenn uns einer darauf hinweist. Dies liegt wohl daran, dass sie uns Halt gibt, Identität, eine Rolle, einen Beruf, gesellschaftliche und persönliche Anerkennung. Wir müssen sogar aufpassen, dass wir nicht zu Fundamentalisten der Psychotherapie werden.

Therapie ist für mich ein Weg der Veränderung, der Überwindung oder Bewältigung von Krankheiten, der Heilung, des Leben Lernens. Psychotherapie ist, wenn wir sie als Wissenschaft betrachten, eine Veränderungswissenschaft. 
Es geht nicht um richtige oder falsche Modelle über die Wirklichkeit, sondern um Veränderungen, um Prozesse und Methoden, die dabei hilfreich sind. Und was hilfreich ist, wird erfahren, gespürt und nicht erdacht. Eine hilfreiche Methode wirkt. Die Wirkung wird gespürt. Der Weg aus der Ideologie heraus ist also ein Weg aus dem Denken heraus zum Spüren. Das Wesentliche wird nicht gedacht oder theoretisch entwickelt, sondern als wesentlich gespürt, gefühlt und erst dann beschrieben. Um von der Ideologie ins Spüren zu kommen ist es zunächst notwendig, die eigene Perspektive, den eigenen Blickwinkel zu relativieren. Dazu ist eine mehrperspektivische Betrachtungsweise hilfreich. Wenn ich oft genug die Perspektiven wechsle und andere Blickwinkel, andere wissenschaftliche oder weltanschauliche Betrachtungsweisen einnehme, dann entsteht in mir eine Fähigkeit zum Perspektivwechsel. Diese Fähigkeit ist nicht mehr in einer Perspektive gefangen. Sie ist davon unabhängig, wird also im Sinne von Jean Gebser (1986) aperspektivisch. Eine solche aperspektivische Haltung ist frei und offen für alle möglichen Informationen, offen für unterschiedliche Blickwinkel, nicht identifiziert oder zumindest fähig, Identifizierung loszulassen, Modelle als Modelle zu sehen und vielleicht auch offen zu sein für etwas völlig Neues. Eine solche Verankerung ermöglicht, nach dem Wesen der Dinge zu fragen, nach dem Wesen der Psychotherapie, nach dem Wesen des Menschseins. Albert Schweitzer schreibt dazu (1999, S. 406):

„Zum Wesen des Denkens gehört, dass es nicht Halt mache, bis es die Fragen des Daseins bis dahin verfolgt hat, wo jede Frage mit den anderen zu der einen großen Frage nach dem Verhältnis meines endlichen Daseins zum unendlichen Dasein zusammenfließt und in ihr aufgeht. Das Denken ist eine Welle, die nicht zur Ruhe kommt, bis sie am Gestade des Unendlichen anschlägt. Keine der Fragen, die das Dasein an mich stellt, ist etwas für sich. Alle sind nur Gestalten, die die große Frage des Verhältnisses meines Seins zum unendlichen Sein annimmt. Nur in ihr offenbaren sie sich dem Wesen nach; nur in ihr sind sie lösbar, sofern sie es überhaupt sind."

Das Wesentliche erschließt sich uns also nicht durch Nachdenken, sondern durch eine Offenheit für das Unendliche, wie Schweitzer es nennt, man könnte auch sagen für den Grund unseres Erlebens, den wir nicht denken, aber spüren können.

Karl Rahner hat ja bekanntlich einmal gesagt:

„Der Fromme von morgen wird ein, Mystiker' sein, einer der etwas, erfahren' hat, oder er wird nicht mehr sein."

Es geht ihm also auch um die Erfahrung jenseits des Glaubens. Vielleicht wird analog dazu der Psychotherapeut von morgen auch ein Mystiker sein, einer der mehr spürt, als seinen Konzepten und seiner Weltanschauung zu folgen. Und dann könnte es analog zu dem heute notwendigen Dialog der Religionen einen echten Dialog der Psychotherapien geben, einen Dialog in Respekt, der die Schönheit und Kompetenz der eigenen Religion oder Schule, aber auch die der anderen würdigt. Seine Basis wäre der konzeptionsfreie Grund unseres Bewusstseins, aus dem heraus sich eine integrierte Psychotherapie entwickeln könnte (s. Abb. 13).

Der wesentliche Schritt liegt also in der Entfaltung unseres Bewusstseins als Therapeuten.

Um dies zu verwirklichen ist es zunächst einmal hilfreich, den eigenen Bewusstseinszustand zu erkennen und ihn dann zu öffnen und zu vertiefen hin zur Erfahrung unserer Seele (siehe den Beitrag „Seele, Geist und kosmische Intelligenz von Joachim Galuska in diesem Buch). 


\begin{tabular}{|c|c|c|}
\hline Fundamentalismus & $\begin{array}{l}\text { Unterwerfung der } \\
\text { anderen Ideologien }\end{array}$ & $\begin{array}{l}\text { „Politik“ der Psycho- } \\
\text { therapie-Verbände }\end{array}$ \\
\hline Ideologie & $\begin{array}{l}\text { Konzeptionelles } \\
\text { Gefangensein }\end{array}$ & $\begin{array}{l}\text { Psychotherapeutische } \\
\text { Schulen }\end{array}$ \\
\hline 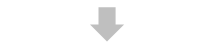 & & \\
\hline Perspektive & Eigener Blickwinkel & $\begin{array}{l}\text { Psychotherapeutische } \\
\text { Kompetenzen }\end{array}$ \\
\hline Mehrperspektivität & Perspektivwechsel & Integrierte Psychotherapie \\
\hline Aperspektivität & Wesen, Seele & „beseelte“ Psychotherapie \\
\hline 1 & & Psychotherapie als \\
\hline Offenheit & Unbekanntes, Leben & $\begin{array}{l}\text { angewandte Bewusst- } \\
\text { seinswissenschaft? }\end{array}$ \\
\hline
\end{tabular}

Abb. 13 Von der Ideologie zur Offenheit

Es gibt verschiedene Zugänge zur Verankerung in unserer Seele, zur Bewusstwerdung unserer Seele. Der direkteste ist wohl der des bewussten Anwesendseins, der Vergegenwärtigung unseres Daseins. Ein weiterer Zugang besteht darin, unsere Aufmerksamkeit von den Inhalten des Erlebens abzuwenden und zu unserem inneren Gewahrsein hinzulenken, also vom Beobachtungsinhalt zum Beobachter, der dann vertieft wird zum sogenannten Zeugen-Bewusstsein. Eine kontinuierliche fließende Achtsamkeit, das Kontinuum unserer Awareness, kann ebenfalls dieses Bewusstsein herstellen. Jede Meditationsmethode ist im Grunde darauf ausgerichtet Bewusstseinsqualitäten zu bewirken, wie Stille, Leere, Weite, Unendlichkeit, Klarheit, Losgelöst sein, Verbundensein usw.

die wir als Kerneigenschaften unserer Seele beschreiben können. Man könnte sich auch unmittelbar auf eine solche reine Bewusstseinsqualität konzentrieren, sich in diese vertiefen oder von ihr ergreifen lassen. Interessant ist, dass jede dieser Qualitäten relativ leicht die Erfahrung anderer solcher Qualitäten eröffnet. Wir können uns also innerhalb dieser verschiedenen Seelenqualitäten bewegen. Da das „Herz“ja nicht ohne Grund auch eine wesentliche Metapher für unsere Seele ist, führt die Öffnung unseres Herzens für uns selbst, für andere Menschen und die Welt im Grunde auch zu einer Berührung und Öffnung unserer Seele.

Wir wirkt sich nun eine solche Seelenverankerung aus in der Form einer „beseelten Psychotherapie“? Wenn ein Verhaltenstherapeut, ein Psychoanalytiker, ein Systemiker oder ein humanistischer Psychotherapeut mit seinem gesamten Wissen und seiner gesamten methodischen Kompetenz her beseelt handelt, begegnet er dem Menschen anders, als wenn er ihn nur als ein psychodynamisches Wesen sieht, das von Triebkräften gesteuert wird, die miteinander in Konflikt stehen, oder wenn er ihn als Patienten sieht, der mit seinen Kognitionen bessere Wege gehen muss oder als ein Klientensystem, das in familiensystemischen Zusammenhängen gefangen ist und dafür neue Lösungen finden muss. Beseelte Psychotherapie ist in diesem Sinne eigentlich keine Technik und keine Methode, sondern sie nutzt Techniken und Methoden und wendet diese beseelt an, handelt beseelt. 


\section{Mehrperspektivische Diagnostik}

Die Verankerung in unserer Seele führt dazu, dass der Therapeut die Identifizierung mit seinen Theorien und Modellen immer wieder lösen kann und damit eine vielschichtige mehrperspektivische Diagnostik betreiben kann, dass er seinen Vorlieben, seinen methodischen und schulenspezifischen Prägungen nicht so ausgeliefert ist und auf diese Weise dem Patienten gerechter werden kann. Und aus dieser Sicht wäre keine der Perspektiven, in denen heute die Psychiatrie oder die Psychotherapie denkt, falsch oder schlecht, sondern sie wären eben immer nur Perspektiven.

Ken Wilber (1996) erläutert, dass wir jedes Phänomen, also auch einen Menschen unter vier Aspekten betrachten können, nämlich sein Inneres und Subjektives, sein Äußeres und Objektives, seinen individuellen autonomen Aspekt und seinen sozialen bezogenen Aspekt. Daraus ergeben sich vier Quadranten oder vier Perspektiven, die die unterschiedlichen Schulen und Richtungen jeweils in ihren Vordergrund stellen:

1. Die individuelle subjektive Welt, das subjektive Erleben, das wir zu verstehen versuchen. Es ist das Feld der tiefenpsychologischen und humanistischen psychotherapeutischen Perspektive.

2. Seine subjektiv soziale Seite, wie er seine Beziehungen erlebt zu seinen Nächsten, zum Therapeuten, aber auch am Arbeitsplatz und innerhalb seiner Kultur. Dies wäre die beziehungsmäßige und sozial-kulturelle Perspektive, etwa der Gruppentherapie und der Paar- und Familientherapie.

3. Das individuell objektive beobachtbare Verhalten des Klienten, auch das Somatische. Es betrifft all das, was sich messen lässt und objektivieren lässt. Es ist die behavioristische und medizinisch-naturwissenschaftliche Perspektive.

4. Sein objektives Sozialverhalten und seine Einbettung im Sozialsystem, wie beispielsweise Arbeitsfähigkeit, Krankheits- und Behandlungskosten, aber auch objektivierbares kommunikatives Verhalten. Das ist die systemisch-soziale Perspektive der Sozialarbeit und der Sozialpsychiatrie.

Während also die diversen „Schulen“ ihren Schwerpunkt in einem dieser Quadranten besitzen oder gar auf ihn fixiert und beschränkt bleiben, ist eine mehrperspek-

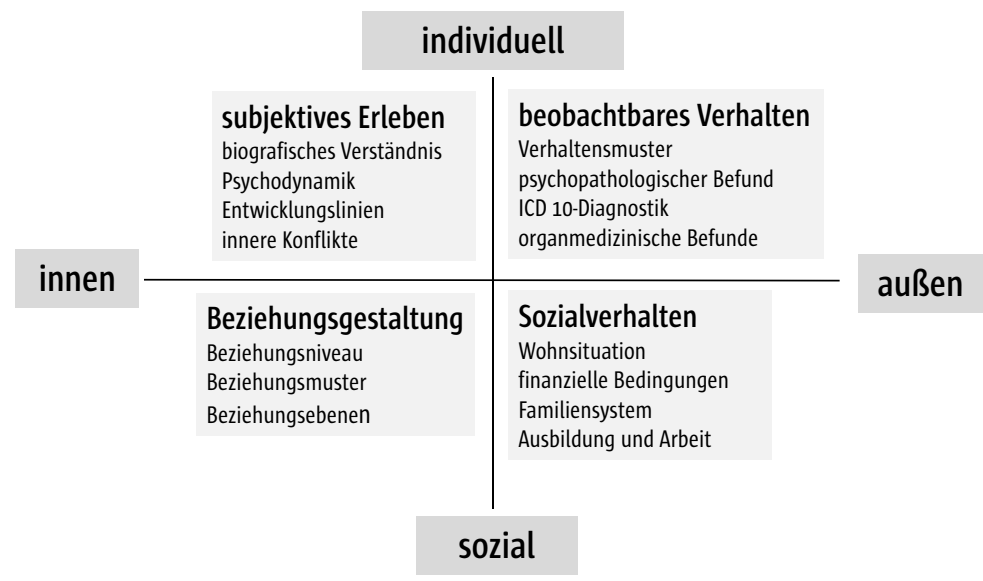

Abb. 14 Integrale Diagnostik 
tivische Diagnostik in der Lage, alle wesentlichen Perspektiven einzunehmen und im Einzelfall zu gewichten.

Sie würdigt also das subjektive Erleben, in das wir uns einfühlen und das wir zu verstehen versuchen, ebenso wie das beobachtbare Verhalten, das zu objektivierbaren Befunden führt, wie seine Art der Beziehungsgestaltung und sein Sozialverhalten (s. Abb. 14).

Das individuelle subjektive Erleben bezieht sich darauf, wie der Patient sich selbst erlebt, sein Leben und seine Probleme versteht, seine Ressourcen und Kompetenzen kennt. Hieraus kann ein biographisches Verständnis entwickelt werden. Eine Erarbeitung der typischen inneren Konflikte ergibt die tiefenpsychologisch bekannte Psychodynamik. Die Diagnostik der individuellen subjektiven Welt kann sich auf alle Entwicklungslinien beziehen. Das Konzept der Entwicklungslinien geht zurück auf Blanck und Blanck (1982) und wurde von Ken Wilber (2001) um einige weitere Entwicklungslinien ergänzt. Neben der Entwicklung des Konzeptes von uns selbst und der Art der Beziehung zu anderen Menschen können insbesondere die Entwicklung der Abwehrfunktionen, die kognitive, die affektive, die moralische Entwicklung und die religiös-spirituelle Entwicklung analysiert und beschrieben werden. Auf jeder dieser Linien machen wir Menschen eine typische Entwicklung durch, die häufig in Stufen oder Phasen erfolgt. Ken Wilber ergänzt die präpersonalen Phasen oder Stufen der Kindheitsentwicklung um die personalen der reifenden Erwachsenenentwicklung und die darüber hinausgehenden transpersonalen Ebenen und Möglichkeiten (s. Abb. 15).

In einer differenzierten Analyse könnte der Entwicklungsstand eines Patienten für jede dieser Entwicklungslinien beschrieben werden .

Die Diagnostik der subjektiv sozialen Seite, also der Beziehungsgestaltung, meint zunächst einmal die Diagnostik des Beziehungsniveaus, das abhängig ist von der entsprechenden Entwicklungslinie der inneren Beziehungsfähigkeit des Patienten. Gestaltet er seine Beziehungen sehr kindlich, jugendlich oder reif? Dies ergibt sich auch aus der Analyse der Beziehungsmuster, seiner typischen Beziehungsgestaltun-

\section{Selbst-Entwicklung \\ (Objekt-)Beziehungen \\ Abwehrfunktionen \\ Kognitive Entwicklung \\ Affekte (u.a. Angstniveau) \\ Moralische Entwicklung \\ Religiös-spirituelle Entwicklung \\ Weitere Linien, z. B. Ästhetik, Bedürfnisse}

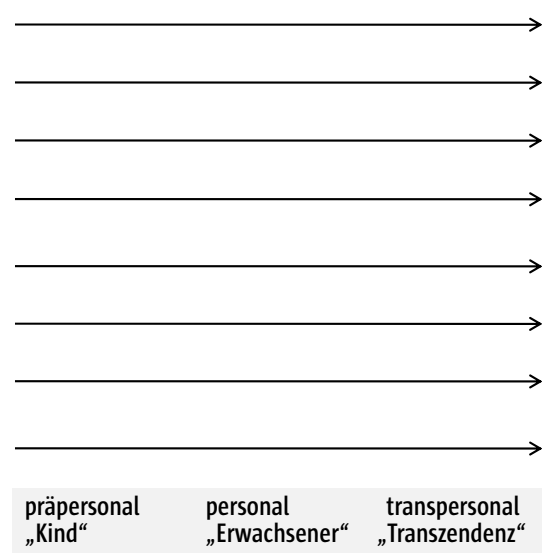


gen. Es ist die Art und Weise, wie er sich selbst und andere in seinen Beziehungen erlebt und wie er von diesen erlebt wird. Die Analyse der Beziehungsebenen meint die Ebenen der therapeutischen Beziehung, die weiter unten beschrieben werden.

Die Diagnostik des individuell beobachtbaren Verhaltens führt aus der Zusammenschau der organmedizinischen Untersuchungsbefunde, der Verhaltensmuster des Patienten und der psychopathologisch relevanten Befunde und Symptome zur Diagnose gemäß der ICD 10 bzw. der DSM 4. Diese diagnostischen Systeme beschreiben ja keine Krankheitseinheiten im engeren Sinne mehr, sondern zunehmend typische Cluster oder Syndrome operationalisierter Symptomkonstellationen.

Das Sozialverhalten des Patienten bezieht sich auf seine Wohnsituation, seine finanziellen Bedingungen, seine Ausbildung, seine Arbeitssituation und seinen Familienstatus. Hier ist auch seine Stellung im Gesundheits- und Sozialsystem zu erläutern.

Eine mehrperspektivische Diagnostik ist somit grundsätzlich offen für eine ganzheitliche Betrachtung. Innerhalb der vier Quadranten könnten sowohl gestörte als auch ungestörte Phänomene beschrieben werden. Neben der üblichen krankheitsorientierten Perspektive könnte also auch eine Gesundheitsperspektive mit Beschreibung der personalen, sozialen und spirituellen Ressourcen und Kompetenzen eingenommen werden.

\section{Therapeutische Haltung}

Das Wesentliche einer über die mehrperspektivische Betrachtung hinausgehenden beseelten Psychotherapie ist die therapeutische Grundhaltung. Sie ist zunächst einmal verankert in der Seele des Therapeuten und offen für das ganze Sein seiner Patienten.

Da ein beseelter Psychotherapeut in der Lage ist, seine Gefühle zu betrachten und auch die, die in ihm durch den Patienten ausgelöst werden, befindet er sich in einer inneren Position jenseits von Übertragung und Gegenübertragung, sodass ihm eine solche Verankerung ermöglicht, auch mit schwierigen Patienten umzugehen, ohne sich mit ihnen zu verwickeln oder sie sich vom Leibe halten zu müssen. Der innere Seelenraum erst lässt die Übertragungsprozesse, also wen oder was dieser Patient in mir sieht, und meine Reaktionen darauf, also die Gegenübertragung, betrachten. Und dies ermöglicht, auch mit heftigen Prozessen wie bei Borderline-Patienten umzugehen.

Diese Funktion, sich in der Offenheit und Präsenz der eigenen Seele zu verankern und in dieser Verankerung im Kontakt mit unseren Klienten zu fließen ist unsere Intuition. Intuition ist meines Erachtens die Kernkompetenz eines Psychotherapeuten, aber auch die Kernkompetenz einer Führungskraft und letztlich die Kernkompetenz eines reifen Menschen, der sein eigenes Leben in der Offenheit für die Welt und das, was sie transzendiert, eben führt und gestaltet. Intuition basiert auf dem offenen Spüren meiner Wirklichkeit und der Wirklichkeit meines Gegenübers. Damit ist nicht das Wahrnehmen von Bauchgefühlen gemeint, sondern das Spüren der inneren Wirklichkeit. Unser übliches alltägliches Ich-Bewusstsein hat Gefühle und Gedanken, denen sich ein anderer beobachtender Teil gegenüberstellen kann. In der Tiefe unserer Seele und unseres Geistes aber spüren und erleben wir, wie sich die eigene innere Welt und die unserer Patienten jeweils anfühlt. Intuitives Fühlen 
schöpft aus der Fülle dessen, was uns bewusst ist, aber auch aus dem, was uns noch unbewusst und unbekannt ist. Intuitives Gespür nutzt unseren Verstand und unsere Modelle und entscheidet, welche brauchbar sind und welche nicht, oder ob wir vielleicht sogar ein neues Modell entwickeln müssen. Intuitiv entscheiden wir als Therapeuten, welches Fachwissen, welche eigenen Erfahrungen, welche persönlichen Reaktionen oder Antworten aus den Tiefen unserer Seele wir angesichts der Komplexität der gegenwärtigen Situation und der Wirklichkeit unserer Patienten ausdrücken. Jede dieser Antworten kann ein neuer kreativer Moment sein, der diesen Menschen berühren, trösten, heilen und weiterbringen kann. Intuition bedeutet innehalten, spüren, die inneren und äußeren Eindrücke verarbeiten, wirken lassen, entscheiden, antworten und wieder innehalten, spüren, wirken lassen, antworten. Intuition ist ein offener wacher Fluss unserer Lebensbewegung. Wir haben sie nicht unter Kontrolle, sondern es ist eher umgekehrt: Sie leitet uns, sie führt uns. Ohne es zu merken leben wir intuitiv unser Leben und handeln letztlich auch intuitiv als Therapeuten. Durch unsere konzeptualisierende Verengung und unsere ideologischen Verhaftungen begrenzen wir ihr Potenzial. Die Wirklichkeit von uns Menschen und damit auch unserer Fachgebiete ist aber wesentlich komplexer, tiefer, weiter, mysteriöser, und darum braucht es eine Befreiung unser Intuition, eine Erweiterung, die unserer Zeit und unserer Würde als Menschen angemessen ist. Eine beseelte und intuitive und in diesem Sinne intelligente Medizin und Psychotherapie wäre wieder eine Heilkunst, die unserer Technologisierung und Evidenzbasierung einen ihr angemessenen Ort zuweist.

\section{Beseelter Therapieprozess}

Das Wesen einer beseelten Psychotherapie besteht darin, dass die Problematik, die Störung, die Krankheit des Patienten in der Seele von uns Menschen aufgehoben wird. Dies bedeutet zunächst, in unserer Seele offen zu sein für das ganze Sein unserer Patienten, für ihren Schmerz, ihr Leid, ihr Schicksal, aber auch für ihre Hoffnungen, ihre Stärke, ihre Größe und ihr Potenzial. Dieses Offensein bedeutet, sich in unserem Inneren von unseren Patienten berühren zu lassen, sie auszuhalten, sie zu ertragen, sie in uns zu tragen, sie in uns wirken zu lassen und dann aus der Tiefe unserer Seele, unserer Stille, unserer Weite, unserer Herzensverbindung, unserer Unberührtheit, unserer Ehrfurcht oder unserer Erschütterung heraus Antworten entstehen zu lassen. Dies sind dann Antworten unserer Seele, die die Seele unserer Patienten ansprechen, ihren eigenen Seelengrund wecken (s. Abb. 16).

Reaktion als Abwehr: Gegenübertragung, „Pathologisierung“, technisches Handeln, Konzepte, Überengagement - Angst

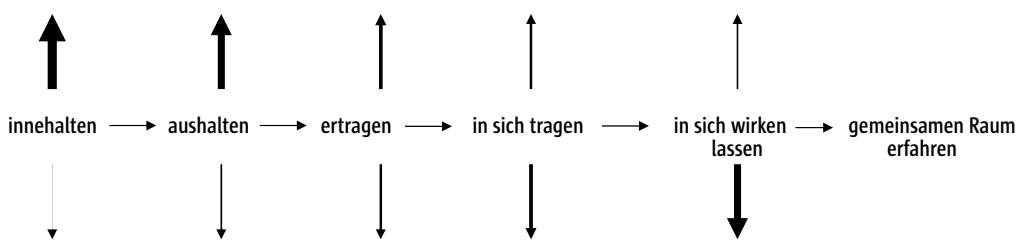

offen und verbunden sein: progressive Impulse, heilende Kraft, Liebe, kreative Idee - keine Antwort 
Die Ausstrahlung der Seele des Therapeuten kann die Seele des Patienten wecken, zum Strahlen und Leuchten bringen und damit ein Gefäß schaffen, einen Kontext für seine Störung, für seine Erkrankung, für sein Schicksal. Die Seelenverbindung innerhalb einer beseelten Psychotherapie kann Heilungs- und Selbstheilungsprozesse in Gang setzen, die aus einer größeren Tiefe und Weite stammen als die unserer Einsichten und Techniken. Wahrscheinlich ist es die Berührung der Ganzheit, Tiefe und Weite unserer Seele mit unseren Verletzungen und Störungen, was das Heilsame ausmacht. Wenn das seelische Überpersönliche und Unendliche also das gestörte Persönliche berührt, kann Heilung geschehen. Eine beseelte Psychotherapie hält diesen Kontext für essenziell und nutzt Methoden und Fachwissen nur in diesem Sinne.

\section{Psychotherapie als angewandte Bewusstseinswissenschaft}

Vertieft sich das Bewusstsein des Therapeuten weiter von der Seele hin zum nondualen Geist (siehe vorhergehender Artikel in diesem Buch), so verwirklicht es das Unbekannte in der therapeutischen Arbeit. Eine so verankerte Psychotherapie ist eigentlich eine angewandte Bewusstseinswissenschaft. Sie stellt sich in den Dienst der Entfaltung des menschlichen Bewusstseins. Ihre Aufgabe besteht im engeren Sinne darin, die Integrität des Bewusstseins wieder herzustellen, wenn Hindernisse, Schwierigkeiten, Blockierungen, Dissoziationen und Desintegrationen auftreten. Eine solche Psychotherapie will nicht nur den einzelnen heilen, sondern fördert auch grundsätzlich die Entfaltung des menschlichen Bewusstseins, indem sie auf Fehlentwicklungen, Verwirrungen und Zerrüttungen des kollektiven Bewusstseins von uns Menschen hinweist. Wir benötigen gegenwärtig eine Psychotherapie, die den Mut hat und bereit ist, uns unserer eigenen Natur zunächst einmal im Nichtwissen, ohne jedes Konzept entgegenzustellen und in der Bereitschaft vor der Unergründlichkeit, Tiefe und Unerfassbarkeit unseres Seins zu leben und eben auch als Therapeuten zu handeln. Nur wenn wir das Leiden unserer Patienten auch in seiner Ursprünglichkeit, Unergründlichkeit und Unerklärlichkeit ertragen können, können wir ihnen erst die Möglichkeit geben, ihr Leben so anzunehmen, wie es wirklich ist, statt sich mit Illusionen und pädagogischen Konzepten darüber zu beschäftigen, wie wir es uns als Therapeuten vorstellen. Die Theorien, die wir über unsere Patienten haben, bilden doch nur die Oberfläche ihrer schicksalhaften Betroffenheit ab. Ihr wirkliches Leben ist etwas viel Unergründlicheres, Tieferes und Fundamentaleres, als wir es uns vorstellen, aber etwas, das wir ständig spüren, dessen Präsenz uns ständig fühlbar ist, dessen Ursprünglichkeit uns ständig andrängt. Auf diesen Grund jenseits aller unserer Konzepte und schulischer Theorien könnten wir uns beziehen. Dieser Grund könnte uns einen, denn wer diesen Grund erfahren hat, spürt, dass es der gemeinsame Grund ist. Er wäre ein Grund für eine erneuerte Psychotherapie, die völlig offen für das Leiden und das Schicksal dieser Menschen ist, die zu uns als Patienten kommen. Dieser Grund ermöglicht uns, ihnen unmittelbar entgegenzutreten und unvoreingenommen diesem Mysterium eines anderen Lebewesens zu begegnen, aus dem auch das Unbekannte spricht. Es geht also darum, eine Offenheit für diese völlig andere, vielleicht zerrüttete Erlebens- und Bewusstseinswelt eines anderen Menschen herzustellen und diese zu realisieren, ohne sie abwehren zu müssen.

Ein im Unbekannten verankertes nonduales Bewusstsein trägt in sich selbst eine innere Freiheit. Es ist frei zu antworten oder auch nicht, etwas in voller Bewusstheit stehen zu lassen oder es einfach verklingen zu lassen. Dies ist eine innerlich souve- 
räne Position zu entscheiden, in welche Perspektive, welches Thema, welches Problem, welche Figur eingetaucht werden möchte, welche wir durchleben wollen und welche nicht. Der nonduale Geist kann sich nicht wirklich verwickeln, da er die Muster erkennt als die, die sie sind. Er besitzt daher eine innere Freiheit innerhalb der Verwicklung, spürt sie, schmeckt sie, ohne an sie gebunden zu sein. Hier entsteht ein gemeinsames Gefühl, eine Wahl zu besitzen, herauszutreten aus den prägenden Mustern, oder sie in einer anderen Richtung weiterzuleben. Vielleicht kann der Patient sich selbst erkennen im Spiegel dieser Bewusstheit als der, der er ist, wie er sich selbst versteht, was ihm selbst wesentlich ist und an welcher Stelle seines Schicksals er eben gerade steht. Wenn in dieser Vergegenwärtigung keine Lösung für die Schicksalsproblematik oder die Zerrüttung der Bewusstseinsstruktur gefunden wird, sucht der nonduale Geist nicht weiter im Fachwissen, der Lebenserfahrung oder dem Raum der Seele, sondern wendet sich dem Unbekannten zu, ist bereit, etwas völlig Neues zu betrachten. Ein so verankertes Bewusstsein kann gemeinsam warten und nicht wissen, ob eine Lösung entsteht. Und wir können es auch annehmen, wenn keine Lösung entsteht, denn dies ist manchmal Schicksal. „Aus dem Ewigen gibt es keinen Ausweg“, sagt Rilke. Im Unbekannten ist alles geborgen, hier löst sich alles auf. Auch die Psychose, auch das absolute Grauen hat hier seinen Platz und ist aufgehoben. Hier kann das Leiden über das Persönliche hinweg erkannt werden als Dukkha, wie der Buddhismus sagt, als Leiden eines einzelnen Menschen, das nicht nur seine persönliche Biographie ist, sondern erfahrenes Leid, das eben Menschen besitzen. Dann ist es nicht länger mein Schmerz, meine Angst, meine Verletztheit, sondern der Schmerz und die Angst, Verletztheit, Zerrissenheit, Krankheit als menschliches Schicksal. Dann kann auch eine tiefere Art von Mitgefühl sowohl bei uns als Psychotherapeuten als auch bei unseren Patienten entstehen, sich selbst und anderen Menschen gegenüber zu treten und unsere Geschichte und unser Leiden als menschliches Leiden zu respektieren, als menschliche Verletzung, als menschliches Schicksal. Das kann dazu beitragen, dass wir uns selbst überhaupt einmal fundamental annehmen und aushalten können, und dies ist angesichts des wahrscheinlich kollektiven Traumatisiertseins von uns Menschen die einzige Haltung, aus der eine Heilung unseres kollektiven menschlichen Schicksals möglich ist. Nahezu jeder von uns trägt in sich selbst Gefühle des Überfordertseins, Überflutetseins, des Verlorenseins im Schmerz, in der Angst, in der Verzweiflung irgendeiner Traumatisierung, um die herum wir allerlei Schutzmechanismen, Abwehrformen und Muster konstruiert haben. Doch diese beherrschen häufig unser Leben, verhindern, dass wir offen aufeinander zugehen können und uns wirklich aufeinander einlassen können. Eine Heilung dieser Traumatisierungen ist nur möglich, wenn das Grauen und der Schmerz, die in diesem inneren Abgrund liegen, erkannt werden und wenn wir realisieren, wie diese Wunde uns prägt und wie wir dies annehmen können als menschliches Schicksal, als Teil des existenziellen Geschehens, dem wir eben ausgeliefert sind. Dieser Realität ins Auge zu schauen, scheint mir nur aus einer so verankerten spirituellen Haltung möglich zu sein, die an kein Muster mehr gebunden ist, sondern im Unbekannten verankert ist, das größer ist als unser Leben und Sterben und eben Teil einer Intelligenz, die wir nicht vollständig begreifen können, die uns aber einen Platz gibt im großen Gefüge der Evolution.

Ein nondualer Geist in der Psychotherapie ist noch nicht einmal daran gebunden, heilen zu müssen, sondern kann auch Krankes krank sein lassen. Unsere Seele möchte natürlich heilen und das Leben schöner machen, da es ihren inneren Werten ent- 
spricht. Manchmal jedoch steht das Heilenwollen dem Heilen im Weg, manchmal ist Nichtberühren besser als Berühren, Lassen besser als Tun, Schweigen besser als Sprechen. Dies zu unterscheiden ist eine Stärke des nondualen Geistes. Manche Künstler, wie Rilke, wären gar nicht so groß und so produktiv geworden, wenn man versucht hätte, sie zu heilen. Vielleicht haben ihre Gestörtheiten und Krankheiten ihr künstlerisches Schaffen angetrieben. Nicht immer ist die Therapie der richtige Weg. Der nonduale Geist kann auch akzeptieren, wenn ein Künstler sagt, dass er nicht gesund werden möchte, weil seine Krankheit ihm Kraft und Inspiration für seine Arbeit gibt. So kann das nonduale Bewusstsein auch in Frieden leben mit der Unheilbarkeit. Selbstverständlich werden wir weiter nach Behandlungsformen schwerer Erkrankungen suchen, aber wir brauchen es nicht als Niederlage zu erleben, wenn wir keine Wege finden. Sondern wir können realisieren, dass dies auch zur Evolution gehört, und es annehmen als Teil des menschlichen Schicksals.

\section{Literatur}

Blanck G, Blanck R (1982) Angewandte Ich-Psychologie. Klett Cotta Verlag Stuttgart

Gebser I (1986) Ursprung und Gegenwart. Novalis Verlag Schaffhausen

Hinderhuber H (2001) Die Seele. SpringerVerlag Wien

Kernberg 0 F (1981) Objektbeziehungen und Praxis der Psychoanalyse. Klett Cotta Verlag Stuttgart

Petzold H (1993) Integrative Therapie. Junfermann Verlag Paderborn

Schweitzer A (1999) Die Weltanschauung der Ehrfurcht vor dem Leben. Kulturphilosophie III, Beck München Wilber K (1996) Eros Kosmos Logos. Krüger Verlag Frankfurt

Wilber K (2001) Integrale Psychologie. Arbor Verlag Freiamt

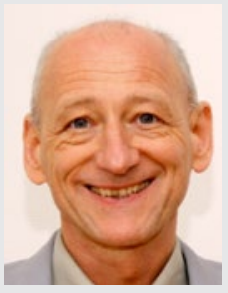

Dr. med. Joachim Galuska

Facharzt für Psychosomatische Medizin und für Psychiatrie und Psychotherapie, Geschäftsführer und Ärztlicher Direktor der Heiligenfeld Kliniken in Bad Kissingen und Waldmünchen. Herausgeber u.a. der Bücher: Die Kunst des Wirtschaftens (2010), Psychotherapie und Bewusstsein (2005), Gründer der Zeitschrift Bewusstseinswissenschaften und der Stiftung Bewusstseinswissenschaften. 